\title{
THE EXTENDED DUST EMISSION AROUND GK PER
}

\author{
S. M. DOUGHERTY ${ }^{1}$, L. B. F. M. WATERS ${ }^{2,3}$, M. F. BODE ${ }^{1}$, \\ H. M. LLOYD ${ }^{1}$, D. J. M. KESTER ${ }^{3}$, TJ. R. BONTEKOE ${ }^{3,4}$ \\ 1. Chemical and Physical Sciences, John Moores University \\ of Liverpool, Byrom Street, Liverpool, L3 3AF, UK \\ 2. Astronomical Institute "Anton Pannekoek", \\ University of Amsterdam, Kruislaan 403, \\ 1098 SJ Amsterdam, The Netherlands \\ 3. SRON Laboratory for Space Research, P.O. Box 800, \\ 9700 AV Groningen, The Netherlands \\ 4. Bontekoe Data Consultancy, Herengracht 47, \\ NL-2312 LC Leiden, The Netherlands
}

GK Per, a classical nova system that erupted in 1901, is one of the more unusual examples of its type. It has the longest known orbital period for a classical nova (1.997d; Crampton, Cowley \& Fisher 1986); and it contains a white dwarf primary with an evolved K2 sub-giant secondary. Most remarkably, the IRAS Sky Survey (1991) reveals that GK Per exhibits far-IR dust emission extending $\sim 17^{\prime}$ to the NW and SE of the nova (Bode et al. 1987; Seaquist et al. 1989). We have re-analysed the IRAS data using maximum entropy reconstruction (Bontekoe et al. 1991; Bontekoe, Koper \& Kester 1994) to resolve structures at a spatial resolution approaching the diffraction limit of IRAS, which is $1^{\prime}$ at $60 \mu \mathrm{m}$ and $1.7^{\prime}$ at $100 \mu \mathrm{m}$.

The analysis of the resulting HIRAS images of GK Per has led us to reconsider the origin of the extended envelope around the binary. For a full discussion see Dougherty et al. (1996). Our conclusions can be summarised as follows:

1. The extended dust emitting region observed by IRAS has a strongly symmetric distribution about the binary. This rules out an interstellar origin. The region is at an approximately uniform temperature of $22 \mathrm{~K}$, which implies the observed discrete emission features are due to density enhancements. The dust mass in the envelope is $0.02 \ldots 0.04 \mathrm{M}_{\odot}$, compared to a gas mass of $1.0 \ldots 1.2 \mathrm{M}_{\odot}$, implying a dust-to-gas ratio of $0.02 \ldots 0.04$. The estimated age of the envelope is $1.110^{5}$ or $1.110^{6} \mathrm{yr}$, dependent on the adopted expansion velocity.

463

A. Evans and J. H. Wood (eds.), Cataclysmic Variables and Related Objects, 463-464.

○ 1996 Kluwer Academic Publishers. Printed in the Netherlands. 
2. The age of the binary compared to the age of the envelope requires that the material in the extended emission region originated from the evolved secondary via Roche lobe overflow to the white dwarf primary, and subsequent ejection by the white dwarf. The initial mass transfer rate was sufficiently high to convert the white dwarf into a 'born-again' AGB star. Thus, the ejection will have taken place in a common envelope phase. The present luminosity and spectral type of the secondary indicate a main sequence mass of $1.3 \ldots 1.5 \mathrm{M}_{\odot}$.

3 . We argue that the discrete emission regions result from multi-epoch mass ejection on a time-scale of 4 or $4010^{4} \mathrm{yr}$. We suggest that they may be the result of thermal pulses of the born-again AGB primary star.

4. The present mass transfer rate and the time that has elapsed since the high mass transfer rate stopped imply that the classical nova outburst in 1901 was the first such event.

5. The shape of the extended emission region strongly suggests a bipolar outflow. CO observations of Scott, Rawlings \& Evans (1994) and the warped appearance of the IR emitting region in the HIRAS images support such a model. However, such a geometry is difficult to understand in the context of common envelope evolution.

The HIRAS images have provided new insight into the evolution of the GK Per binary system. To verify the model outlined above, accurate determination of the expansion velocity of the extended region and improved binary parameters are required. In addition, the detailed three-dimensional geometry of the extended emission region needs to be clarified, which we aim to achieve via mapping of the $\mathrm{CO}$ and $\mathrm{H}$ I line emission. The smallscale structure of the emission region will be a target for the Infrared Space Observatory (ISO).

\section{References}

Bode M.F., Seaquist, E.R., Frail, D.A., et al., 1987, Nature, 329, 519

Bontekoe, Tj.R., Kester, D.J.M., Price, S.D., et al., 1991, A\&A, 248, 328

Bontekoe, Tj.R., Koper, E., Kester, D.J.M., 1994, A\&A, 284, 1037

Crampton, D., Cowley, A.P., Fisher, W.A. 1986, Ap. J., 300, 788

Dougherty, S.M., Waters, L.B.F.M., Bode, M.F., et al., 1996, A\&A, in press

IRAS Sky Survey Atlas Explanatory Supplement, 1991, Wheelock S., Gautier T.N., Chillemi J., et al., (eds.)

Scott, A.D., Rawlings, J.M.C., Evans, A., 1994, MNRAS, 269, 707

Seaquist, E.R., Bode, M.F., Frail, D.A., et al., 1989, Ap. J., 344, 805 